\title{
Solution of the problem of negative value of the linear technological size
}

\author{
Vasilii Masyagin ${ }^{1, *}$, Ruslan Bazhenov ${ }^{2}$, and Andrei Muholzoev ${ }^{3}$ \\ ${ }^{1}$ Omsk State Technical University, Mira 11, Omsk 644050, Russia \\ ${ }^{2}$ Sholom-Aleichem Priamursky State University, Shirokaya 70a, Birobidzhan 679015, Russia \\ ${ }^{3}$ National Research Tomsk Polytechnic University, Lenina 30, Tomsk 634050, Russia
}

\begin{abstract}
The calculation of the linear technological sizes is one of necessary design stages of technological processes of machining. A problem is that with performance of calculation of the linear technological sizes negative value of the technological size sometimes turns out. In the article consideedr an example of calculation, in which appears negative value of the technological size. The calculation is carried out on the technique with use of the chart of processing and the computer program which is carrying out automatic construction and the decision of the equations of dimension chains. As a result of the study, it was found that the appearance of a negative value when calculating the technological dimensions is not an error, this is due to the uncertainty of the positions of the technological size boundaries before calculation.
\end{abstract}

\section{Introduction}

The calculation of the technological sizes is one of necessary design stages of technological processes of machining. A theoretical basis of calculation is the theory of dimensional chains or tolerance charting, mathematical model is graph $[1,2,3,4,5]$. Nowadays given calculation is automated [6].

A problem is that with performance of calculation of the linear technological sizes negative value of the technological size sometimes turns out.

On the sense the technological size is always positive, so it is necessary to understand what is the cause of the negative value of the technological size, and what needs to be changed when obtaining a negative value of the technological size as a result of calculation.

\section{The description of the research}

Consider an example of calculation, in which there are negative value of the technological size. The calculation is carried out on the technique of Mordvinov [1] with use of the computer program which is carrying out automatic construction and the decision of the equations of dimension chains.

Let it is necessary to manufacture a detail (Fig. 1), having the linear design dimensions. The sketch of the workpiece and technological sketches with unknown linear technological dimensions are shown in Fig. 2 and Fig. 3.

The unknown technological dimensions are designated by a symbol $A_{i j}$, where the indexes designate the sequence number of the operation and the sequence number of the technological size.

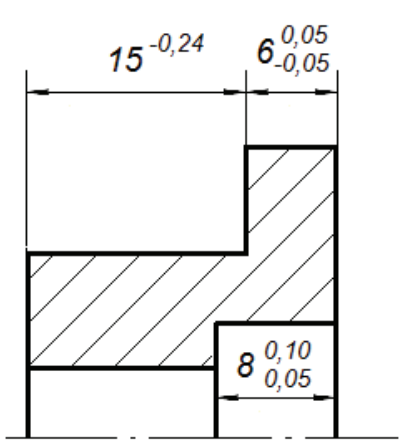

Fig. 1. The sketch of detail with linear design dimensions.

Op. 0

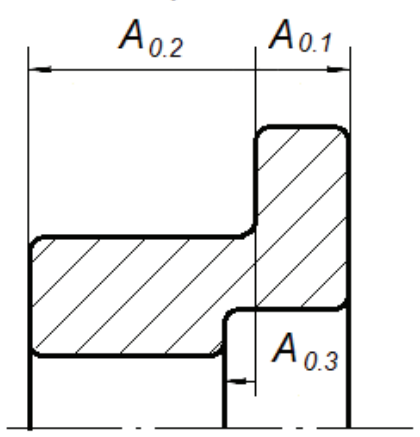

Fig. 2. The drawing of workpiece with linear technological dimensions.

\footnotetext{
* Corresponding author: masyagin@omgtu.ru
} 
Op. 1

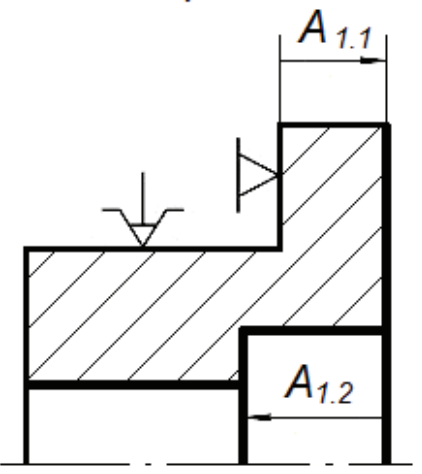

Op. 2

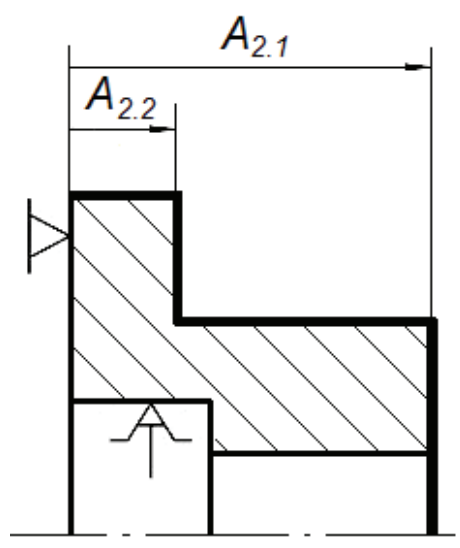

Op.3

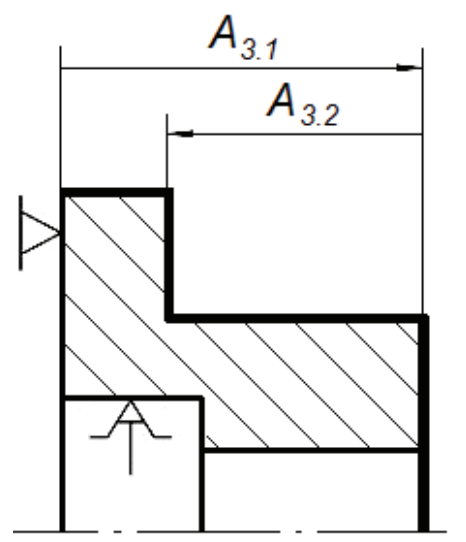

Op.4

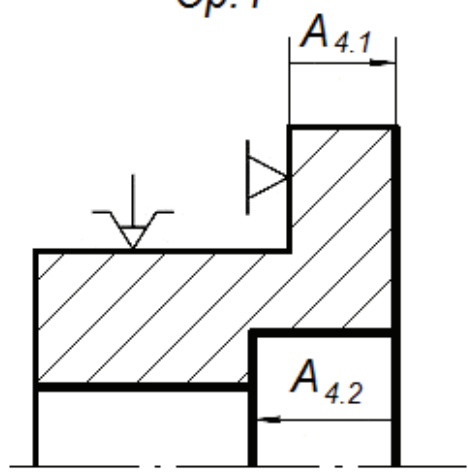

Fig. 3. The technological sketches with linear technological dimensions.
The technological dimensions are shown by onesided arrows pointing towards the surfaces to be treated.

It should be noted that, according to the technological recommendations, all dimensions of the workpiece must be assigned from a processing base at the first operation (Fig. 3).

According to the sketches of the part, workpiece and technological sketches, the processing scheme is executed (Fig. 4). The formation of the processing scheme is reduced to the transfer of technological dimensions from the technological sketches and design dimensions from the sketch of the part to the contour of the workpiece.

The chart of processing of the linear technological sizes is shown in a Fig. 4. The chart of processing contains all the technological dimension chains with concluding links - the design sizes and the allowances, and component links - the technological sizes.

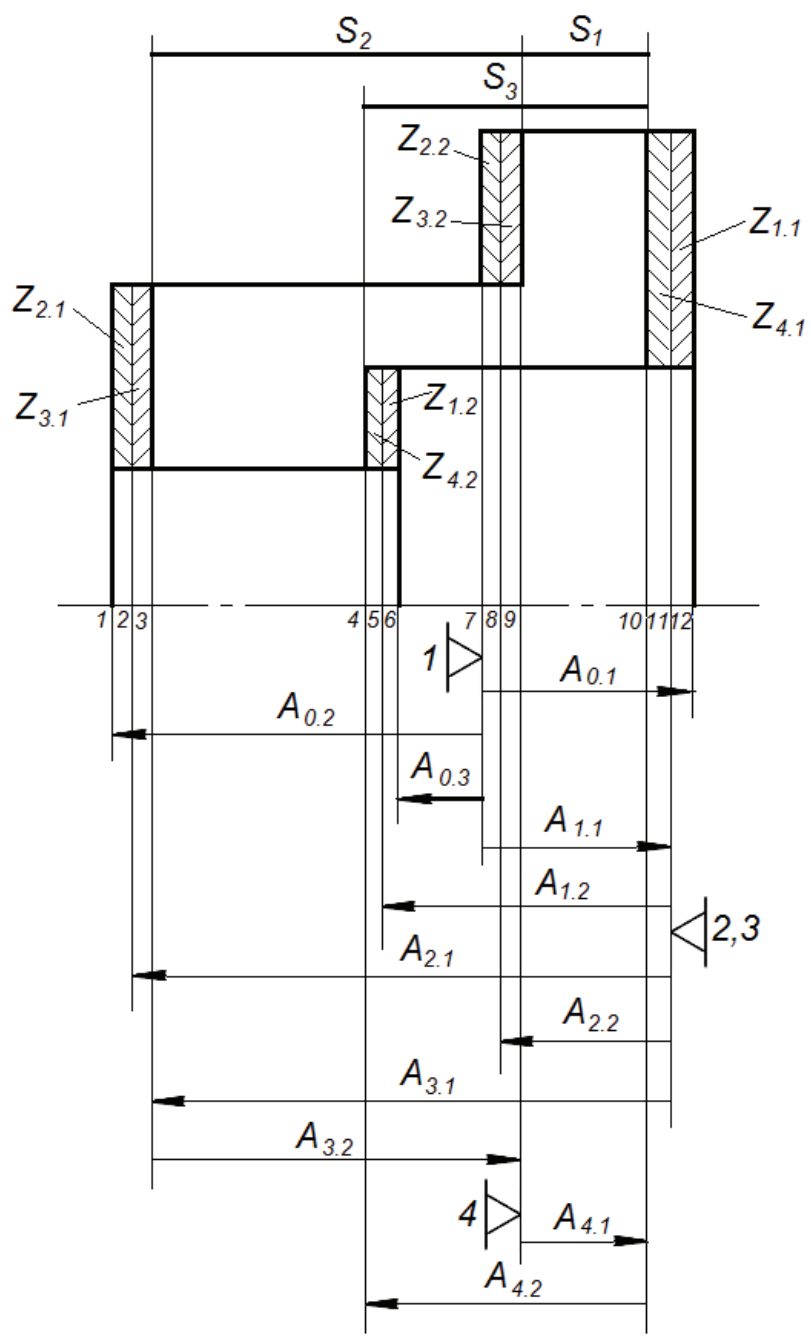

Fig. 4. The initial chart of processing.

The design sizes are designated by a symbol $S_{i}$ with an index designating a sequence number of the design size.

The allowances are denoted by the symbol $Z_{i j}$ with the index corresponding to the index of the technological size $A_{i j}$, according to which the allowance is removed. 
The technological dimensions $A_{i j}$ have the same designation as in Fig. 2 and Fig. 3.

The base surfaces are designated by a special symbol with the indication of the sequence number of operation.

All processable and processed surfaces are numbered from left to right in the growing order.

The values of deviations of the technological sizes and the values of the minimal allowances are determined by handbooks.

The constructed processing scheme must satisfy following conditions [1].

1. Only one arrow of technological size should indicate each surface, and no arrows should indicate the source base. In this example scheme satisfy the condition.

2. The number of technological dimensions, including the dimensions of the workpiece, should be equal to the sum of the quantities of design dimensions and allowances (excluding allowances, the removal of which changes the shape of the workpiece). In this example: 11 sizes of $A_{i j}, 3$ sizes $S_{i}$, and 8 allowances $Z_{i j}$. Therefore, $11=3+8$. This satisfies the condition.

3. The number of technological dimensions, as well as the number of design dimensions and allowances should be one less than the number of surfaces. In this example, 12 surfaces and 11 sizes. This satisfies the condition.

The further calculation are carried out with use of the computer program.

The initial data for computations is given in Table 1 and Table 2.

Table 1 and Table 2 was formed according to the scheme presented in Figure 4.

Table 1. Technological dimensions.

\begin{tabular}{|c|c|c|c|c|}
\hline \multirow{2}{*}{$\begin{array}{c}\text { Index of } \\
\text { size }\end{array}$} & \multirow{2}{*}{$\begin{array}{c}\text { Upper } \\
\text { deviation }\end{array}$} & $\begin{array}{c}\text { Lower } \\
\text { deviation }\end{array}$ & \multicolumn{2}{|c|}{ Boundary } \\
\cline { 4 - 5 } & 0.450 & -0.450 & 7 & 12 \\
\hline 0.1 & 0.550 & -0.550 & 1 & 7 \\
\hline 0.3 & 0.300 & -0.300 & 6 & 7 \\
\hline 1.1 & 0.180 & -0.180 & 7 & 11 \\
\hline 1.2 & 0.180 & -0.180 & 5 & 11 \\
\hline 2.1 & 0.260 & -0.260 & 2 & 11 \\
\hline 2.2 & 0.180 & -0.180 & 8 & 11 \\
\hline 3.1 & 0.105 & -0.105 & 3 & 11 \\
\hline 3.2 & 0.090 & -0.090 & 3 & 9 \\
\hline 4.1 & 0.050 & -0.050 & 9 & 10 \\
\hline 4.2 & 0.025 & -0.025 & 4 & 10 \\
\hline
\end{tabular}

Table 1 contains the following data.

Column 1 is a consecutive list of designations for all linear technological dimensions $A_{i j}$, starting from the dimensions of the workpiece and ending with the technological dimensions obtained at the finish operations.

Column 2 contains an upper tolerance deviation for the technological size with the corresponding sign.

Column 3 contains a lower tolerance deviation for the technological size with the corresponding sign.

Columns 4 and 5 contain, for each technological measurement, the numbers of surfaces (boundaries) that bound the size to the left and the right in accordance with the part processing scheme.

Table 2 contains the following data.

Column 1 contains a sequential list of designations for all of the design dimensions of the detail $-S_{i}$, then allowances for processing $-Z_{i j}$.

Column 2 contains the nominal dimensions of the detail according to the drawing are indicated (Fig. 1) and the minimum $Z_{\min }$ values of allowances, according to the handbooks.

Columns 3 and 4 contain the upper and lower deviations in the dimensions of $\mathrm{Si}$, in accordance with the drawing of the part (see Fig. 1). For allowances, zero values are entered in both columns.

Columns 5 and 6 contains, for each design dimensions of the detail and for each allowance the numbers of the surfaces (boundaries) that bound the size or allowance to the left and to the right according to the part machining scheme are indicated.

Table 2. Design sizes and allowances.

\begin{tabular}{|c|c|c|c|c|c|}
\hline $\begin{array}{c}\text { Index } \\
\text { of } \\
\text { size }\end{array}$ & Nomina & $\begin{array}{c}\text { Upper } \\
\text { I size }\end{array}$ & $\begin{array}{c}\text { Loviatio } \\
\mathbf{n}\end{array}$ & $\begin{array}{c}\text { Lower } \\
\text { deviation }\end{array}$ & \multicolumn{2}{|c|}{ Boundary } \\
\hline 1 & 6.000 & 0.050 & -0.050 & 9 & 10 \\
\hline 2 & 15.000 & 0.000 & -0.240 & 3 & 9 \\
\hline 3 & 8.000 & 0.100 & 0.050 & 4 & 10 \\
\hline 1.1 & 0.490 & 0.000 & 0.000 & 11 & 12 \\
\hline 1.2 & 0.490 & 0.000 & 0.000 & 5 & 6 \\
\hline 2.1 & 0.490 & 0.000 & 0.000 & 1 & 2 \\
\hline 2.2 & 0.490 & 0.000 & 0.000 & 7 & 8 \\
\hline 3.1 & 0.200 & 0.000 & 0.000 & 2 & 3 \\
\hline 3.2 & 0.200 & 0.000 & 0.000 & 8 & 9 \\
\hline 4.1 & 0.200 & 0.000 & 0.000 & 10 & 11 \\
\hline 4.2 & 0.200 & 0.000 & 0.000 & 4 & 5 \\
\hline
\end{tabular}


Table 3. Calculated technological dimensions.

\begin{tabular}{|c|c|c|c|}
\hline Index of size & $\begin{array}{c}\text { Upper } \\
\text { deviation }\end{array}$ & $\begin{array}{c}\text { Lower } \\
\text { deviation }\end{array}$ & $\begin{array}{c}\text { Nominal } \\
\text { size }\end{array}$ \\
\hline 0.1 & 0.450 & -0.450 & 15.000 \\
\hline 0.2 & 0.550 & -0.550 & 10.000 \\
\hline 0.3 & 0.300 & -0.300 & -2.150 \\
\hline 1.1 & 0.180 & -0.180 & 8.500 \\
\hline 1.2 & 0.180 & -0.180 & 7.500 \\
\hline 2.1 & 0.260 & -0.260 & 22.000 \\
\hline 2.2 & 0.180 & -0.180 & 7.500 \\
\hline 3.1 & 0.105 & -0.105 & 21.350 \\
\hline 3.2 & 0.090 & -0.090 & 14.900 \\
\hline 4.1 & 0.050 & -0.050 & 6.000 \\
\hline 4.2 & 0.025 & -0.025 & 8.075 \\
\hline
\end{tabular}

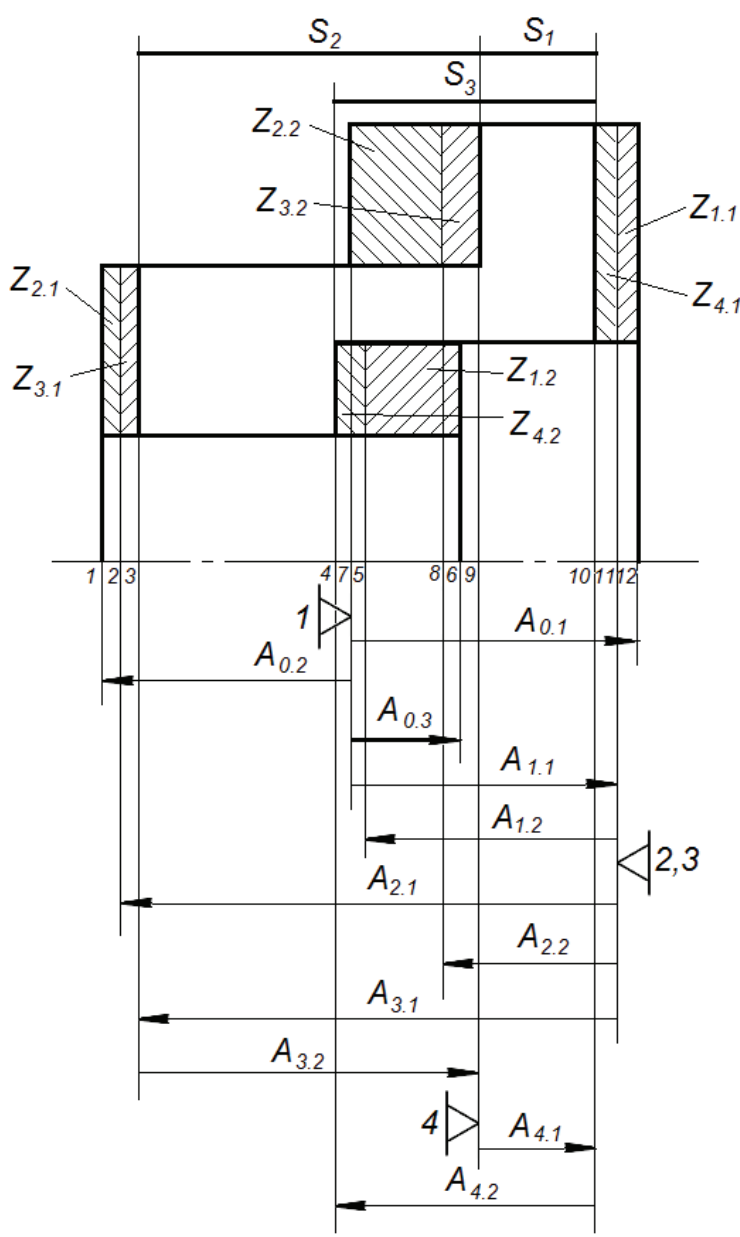

The results of computations is given in Table 3 .

In Table 3, the column "Nominal size" contains the required values of technological parameters, which, with the corresponding upper and lower deviations, are inserted in the technological documentation.

As a result of the computations the negative value of the technological size for one of the sizes of workpiece $A_{0.3}$ turns out (-2.150).

Based on the calculation results, a new processing scheme was implemented (Fig. 4). In this processing scheme, the position of the boundaries is determined by the nominal values of the linear technological dimensions.

The actual position of the left and right boundaries of the size A0.3, as seen from Fig. 5, turned out to be the opposite to the position that was originally taken before the calculation, in accordance with the scheme in Fig. 4.

The given size A0.3 as a result of computations has negative value. Hence, the occurrence of negative value of the technological size is connected to change of real position of the left and right boundaries of the size in relation to initial.

From here follows, that the given size A 0.3 should be designated in another way on the drawing of workpiece (Fig. 2), namely, as shown in the chart of processing (Fig. 5).

\section{Conclusion}

The result of calculating the process dimensions with obtaining a negative value is not an error, but is associated with the uncertainty of the positions of the size boundaries before calculation.

The actual positions of the size boundaries obtained as a result of the calculations may differ from the accepted positions of the boundaries before calculation.

When a negative value is obtained, it is necessary to change the positions of the boundaries of the corresponding technological size in the technological documentation.

\section{References}

1. B.S. Mordwinov, Feingerätetechnik, 24(5), 215-218, (1975)

2. K.A. Ngoi, C.C. Kai, Int. J. of Adv. Manuf. Technol., 8, 175-181, (1993)

3. B.K.A. Ngoi, O.C. Teck, Int. J. of Adv. Manuf. Technol., 8, 371-376, (1993)

4. G.A. Britton, G. Timm, Int. J. of Adv. Manuf. Technol., 20, 448-453, (2002)

5. G. Thimm, G.A. Britton, S.C. Fok, Int. J. of Adv. Manuf. Technol., 24, 261-271, (2004)

6. P. Ji, X. Jianbin, Proceedings of the Institution of Mechanical Engineers, 220, 883-892, (2006)

Fig. 5. The resulting chart of processing. 